\title{
Impact of an organizational structure on the resilience of production processes based on artificial factors in the chemical industry
}

\author{
Hajime Eguchi $^{\mathrm{a},{ }^{*},}$,Tomomi Aoyama ${ }^{\mathrm{a}}$, Kohei Seki ${ }^{\mathrm{a}}$, Donal O’Donovan ${ }^{\mathrm{b}}$, Ichiro Koshijima ${ }^{\mathrm{a}}$ \\ ${ }^{a}$ Nagoya Institute of Technology, Gokiso-cho, Showa-ku, Nagoya-city, Aichi-ken, 466-8555, Japan \\ ${ }^{\mathrm{b}}$ Cork Institute of Technology, Rossa Avenue, Bishopstown, Cork, Ireland \\ * Corresponding author: h.e@ triton.ocn.ne.jp
}

\begin{abstract}
The production process in the chemical industry should be resilient against the influence of disruptive signals introduced into, and emanating from, the production process. Resilience is necessary to maintain high productivity, and is produced by (a) the skills and knowledge of the production staff (human factors), (b) the production units and the production support system (PSS, The computer system to support the production) (artificial factors). In order to maintain the higher resilience produced by the artificial factors, such as a production unit or a PSS, it is critical for the managerial objectives to organize effectively the managerial components, identified as the key element by the organization. In this paper, the aspects under consideration are the managerial components of the resilience matrix classified by the scale of disruptive signal, the managerial objectives used to improve the resilience by the artificial factors in the production process, the specific approaches adopted to manage these artificial factors, and some concluding remarks regarding the managerial objectives. The production unit cannot cope with a large disruptive signal that has not occurred yet, and the skills and knowledge of the human factors are the only one countermeasure against this kind of disruptive signal. The PSS copes with the small or medium disruptive signal instead of the human factor, and it yields the margin of work hours to practice the training and education for the improvement of the skills and knowledge of the production staff.
\end{abstract}

Keywords: Chemical industry, Resilience, Production process, Artificial factors, Disruptive signal.
There is a large variety of disruptive signals that affect, or originate from the production process. These signals often influence or disturb the operation of the production process itself (See Fig.1). The concept of resilience is defined as the capability to restrain the influences of a disruptive signal ${ }^{(1,2,3)}$. It is possible to classify a disruptive signal by scale, and by response provider, who is in charge of the organizational measures necessary to manage the resilience, i.e. individual, group and/or organization ${ }^{(4)}$. The authors of this paper have already discussed the managerial components to restrain the disruptive signal, i.e. to improve resilience based on human factors ${ }^{(5)}$. The principal elements of a production process and the source of resilience are: (a) the skills and knowledge of the production staff (i.e. human factors), (b) the production units and the production support system, hereafter, referred to as PSS (i.e. artificial factors). This paper discusses the managerial components, the managerial objectives and the specific approaches, that are necessary to maintain and improve resilience by the artificial factors in the production process. Only the human factors cope with a large disruptive signal that has not occurred yet. The PSS can deal with the small or medium disruptive signal instead of the human factors, and the margin of work hours yielded in the daily routine of the production staff will be devoted to the training and education to improve the skills and knowledge. So, the artificial factors in the production process have to contribute to draw out the capability of production staff.

\section{Introduction}




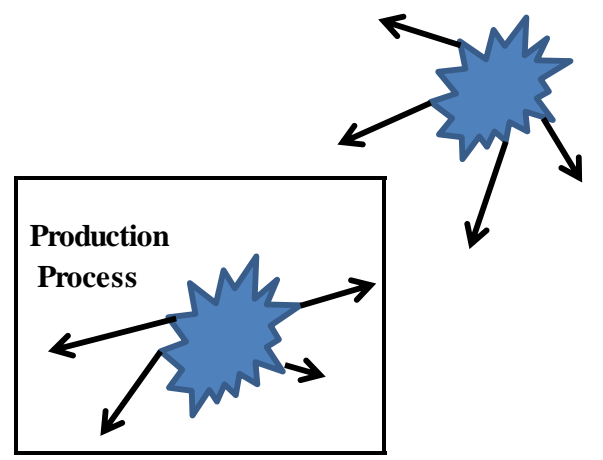

1) Internal

Set point change

Sequence control

Emergency stop

Start up / Shut down

Signal

2) External

Natural desaster

Order from clients

Dis ruption

Fig.1 Disruption inside and outside a production process

\section{Artificial factors that yield resilience in the production process}

The production units and the PSS (artificial factors) are the elements of the production process. Each contributes its own resilience to restrain various kinds of disruptive signals. The production unit has an initial resilience to resist the assumed disruptive signals, but the initial resilience decreases with the deterioration of equipment, or with a disruption that was proved larger than that initially assumed. In this context, the managerial subjects observe and handle the managerial objectives (See Fig.2). In the resilience matrix designed to manage resilience ${ }^{(4,5)}$, the vertical axis corresponds to the scale of disruptive signal, and the horizontal one corresponds to the managerial objectives presided by the managerial subjects. The managerial components of production units are shown in Fig.3. As the production units do not possess the ability to recover the deteriorated resilience by themselves, it is necessary to estimate resilience through the managerial components proposed in Fig.3, and to apply a suitable measure to maintain and improve resilience in accordance with the management policy of the company. Likewise, the managerial components of the PSS are shown in Fig.4. There are two categories of disruptive signals: (a) the disruptive signal that has already occurred, and whose degree of influence is known; (b) the disruptive signal that has not yet occurred, and whose degree of influence is not known.

\section{Resilience management of production units}

The vertical entries of the resilience matrix consist of the managerial components, organized in terms of increasing disruptive signal. The horizontal entries represent the managerial objectives i.e. inspectional patrol, occurrence of trouble, rebuilding and repair of a unit, and risk assessment. For the horizontal entries, the left hand side deals with the disruptive signals that have already occurred, while the right hand side is concerned with the disruptive signals that have not yet occurred.

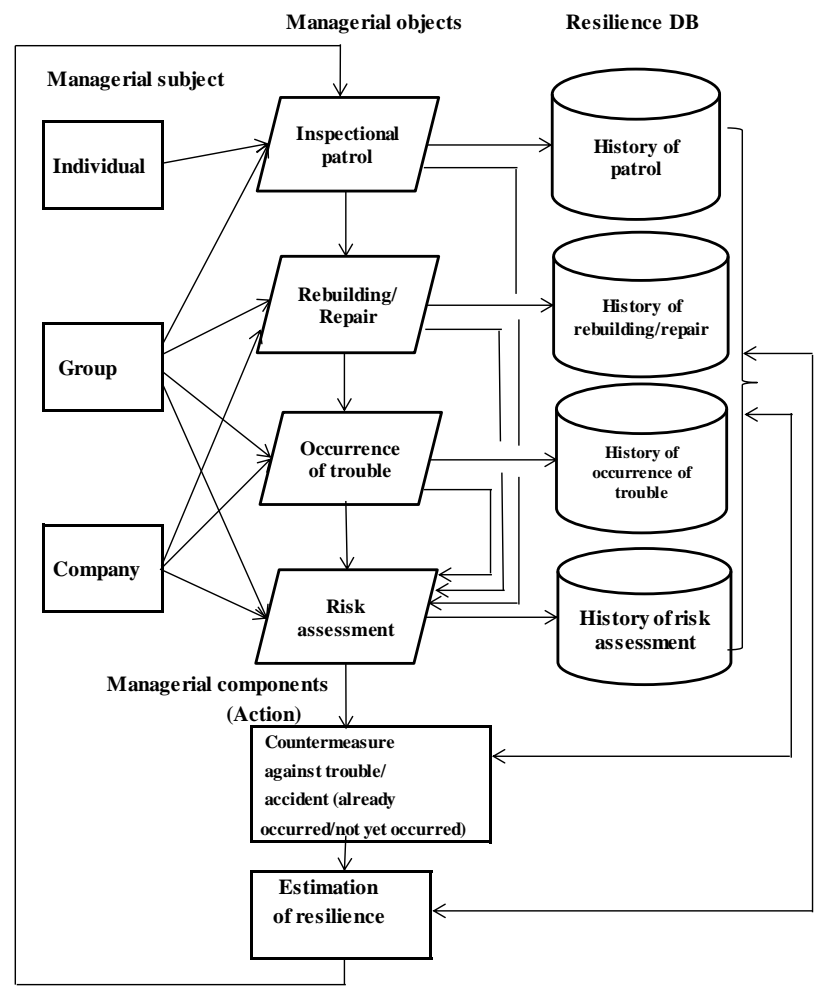

Fig.2. Managerial subjects and objects (production units)

(a) In the case that the disruptive signal has already occurred:

-Inspectional patrol: the inspectional patrol is a daily routine in the production process. The production staff detects the situation of trouble using the human five senses, and detects the threat of an accident by the inspection instruments. The detection of a symptom (a symptom is also disruption) of a large scale trouble is also the target of patrol.

-The occurrence of trouble: the occurrence of trouble suggests the decline of resilience. In this case, a quick repair is required. As the potential risk of similar trouble is often found in the history of troubles in the production process, the risk assessment is always 
necessary after the occurrence of trouble.

(b) In the case that the disruptive signal has not yet occurred :

-Rebuilding and repair of units: units are rebuilt to change the products or the specifications of products, and they are repaired according to the necessity. Rebuilding and repair influence the resilience of the units, therefore, once they have been completed, risk assessment is necessary.

- Risk assessment: risk assessment identifies the degree of the potential risk associated with a particular unit, and its results are used to estimate the resilience of the production process.

\subsection{Managerial components to manage the resilience of production units}

The managerial objectives, managed by the managerial subjects, are achieved by means of the managerial components that depend on the scale of disruptive signal. Each managerial component is referred to as a "Step".

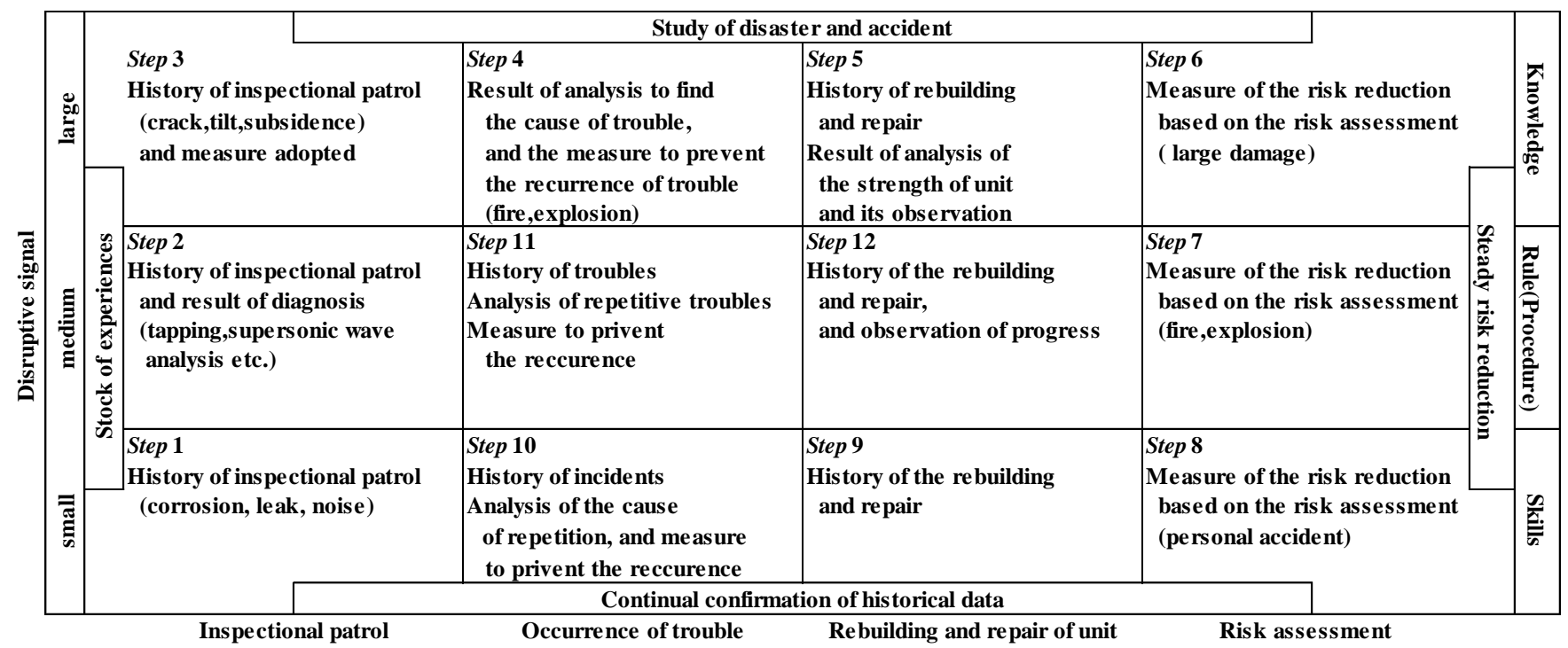

Fig. 3. Managerial components of production units

Step 1 represents the managerial components of resilience used to counter a small disruptive signal found during an inspectional patrol. The production staff often detects a corrosion or leak of materials, or noise caused by a break of the equipment. The rebuilding or repair is done soon after the trouble detection. The managerial component is the history of the inspectional patrol.

Step2 represents the managerial components of resilience used to counter a medium disruptive signal found during an inspectional patrol. If the visual detection of trouble is difficult, the production staff diagnoses the status of the equipment by means of the tapping or using the supersonic wave analyzer etc. The managerial components are the history of the inspectional patrol and the result of diagnosis.

Step3 represents the managerial components of resilience used to counter a large disruptive signal found during an inspectional patrol. Occasionally, the production staff detects a crack, a tilt or subsidence of the equipment.
The repair of these troubles is a matter of urgency, as it may provoke a larger accident, an outflow of dangerous and toxic materials, or a collapse of the structure during a natural disaster. The managerial components are the history of the inspectional patrols and the measures adopted.

Step4 represents the managerial components of resilience used to counter a large disruptive signal at the occurrence of trouble. The disruptive signal might result in the occurrence of a crack, a tilt, a subsidence, a fire, an explosion or a serious personal accident. The managerial components are the result of analysis to find the cause of trouble, and the measure to prevent the recurrence of similar trouble. In this case, rebuilding or repair is necessary.

Step5 represents the managerial components of resilience used to counter a large disruptive signal after the rebuilding and repair of a unit. The rebuilding of units caused by a change of product or by the occurrence of 
trouble, and the repetition of repairs of equipment often accelerates the decline of the strength and/or the endurance of the units, and increases the potential risk. The managerial components are the history of the rebuilding and repair, the result of analysis of the strength of unit and its observation after the rebuilding and repair.

Step6 represents the managerial components of resilience used to counter a large disruptive signal, found by the risk assessment, for which the assumed damage is very large. . The managerial components are the measure of a risk reduction based on the risk assessment.

Step 7 represents the managerial components of resilience used to counter a medium disruptive signal found by the risk assessment. In this case, the fire or the explosion is identified as the hazard by the risk assessment. The managerial components are the measure of the risk reduction based on the risk assessment.

Step 8 represents the managerial components of resilience used to counter a small disruptive signal found by the risk assessment. In this case, the personal accident is identified as the result of the risk assessment. The managerial components are the measure of risk reduction based on the risk assessment.

Step 9 represents the managerial components of resilience used to counter a small disruptive signal after the rebuilding and repair of a unit. In this case, the small repair is carried out according to the result of inspectional patrol, the occurrence of the trouble or the results of a risk assessment. The managerial components are the history of rebuilding and repair of the unit.

Step 10 represents the managerial components of resilience used to counter a small disruptive signal at the occurrence of trouble. In this case, the signal is a small incident, i.e. near-miss or slight trouble that often recurs in the production process. The managerial components are the history of incidents, the analysis of the cause of the recurrence, and the measures introduced to prevent the recurrence of the incident.

Step 11 represents the managerial components of resilience used to counter a medium disruptive signal at the occurrence of trouble. In this case, the recurrence of trouble appearing as a medium disruptive signal causes more serious trouble. So, the managerial components are the history of the troubles, the result of analysis of repetitive troubles and the measure to prevent the recurrence of the trouble.

Step 12 represents the managerial components of resilience used to counter a medium disruptive signal after the rebuilding and repair of a unit. In this case, the repetitive rebuilding and repair in the same part of a unit occasionally results in more serious trouble. So, the managerial components are the history of the rebuilding and repair of the unit and the result of observation of progress.

\subsection{Specific approaches and some remarks about managerial objectives}

(a) Inspectional patrol

-It is critical that the production staff executes the inspectional patrol correctly, identifying the inspectional items at risk for each equipment and their potential to cause trouble.

- The production staff must endeavor not to miss any troubles and make the most of five senses during the inspection.

-If any trouble is detected, the production staff must inform the team members of the situation as soon as possible. Should the supervisor deem it necessary, he would convey the information to another group in the company and notify the appropriate public authority.

(b) Occurrence of trouble

-At the occurrence of trouble, its cause must be analyzed completely, and shared with another group in the company.

-If the trouble recurs frequently in the same part of a unit, it may be a symptom of a more serious underlying problem. In this case, further action, more fundamental than a simple repair, is required. No matter how small the trouble is, an analysis of the problem is necessary, and any corrective conclusion drawn should also be applied to other similar units.

(c) Rebuilding and repair of units

-A detailed history of rebuilding and repair must be preserved and arranged to be available to the company as a whole.

- After the rebuilding, it is necessary to confirm periodically that the operation of units conforms to the specification.

-In case of recurrence of repair, the replacement of a unit should be considered.

(d) Risk assessment

- The risk assessment of a production process should be carried out periodically, and if the result of the assessment changes, special care should be taken. 
-If the level of risk is too high, the measures to reduce the risk should be executed in accordance with the managerial plan.

- The result of the risk assessment of a production process must be shared with other production processes.

\section{Resilience management in the production support system (PSS)}

It is possible to summarize the managerial components, used to manage the resilience achieved by the production support system (PSS), by means of the resilience matrix in Fig.4. In this matrix, the vertical entries are organized according to the scale of the disruptive signal, and the horizontal ones are (a) basic data gathering and alarm function; (b) operational function, based on the operational skills of production staff that mainly require the use of hands and legs; (c) memory function, based on the memory skills of production staff that require the use of memory for the smooth operation of a production process; (d) communication function, based on the communication skills of production staff that require the mutual verbal communication. Except for (a), the classification corresponds to the category of production staff's skills ${ }^{(7)}$, and as to the level of skills: (d) is higher than (c), and (c) is higher than (b). In this section, a large disruptive signal may be interpreted as a signal that has no rules (i.e. procedures) and no skills to deal with it; a medium disruptive signal may be interpreted as a signal that occurs irregularly, for which rules and procedures exist; finally a small disruptive signal may be interpreted as a signal that occurs regularly and has rules and skills associated to it. The managerial subjects, individuals, groups and company, are equally concerned with the managerial objectives in the case of PSS.

\subsection{Managerial components to manage the resilience of PSS}

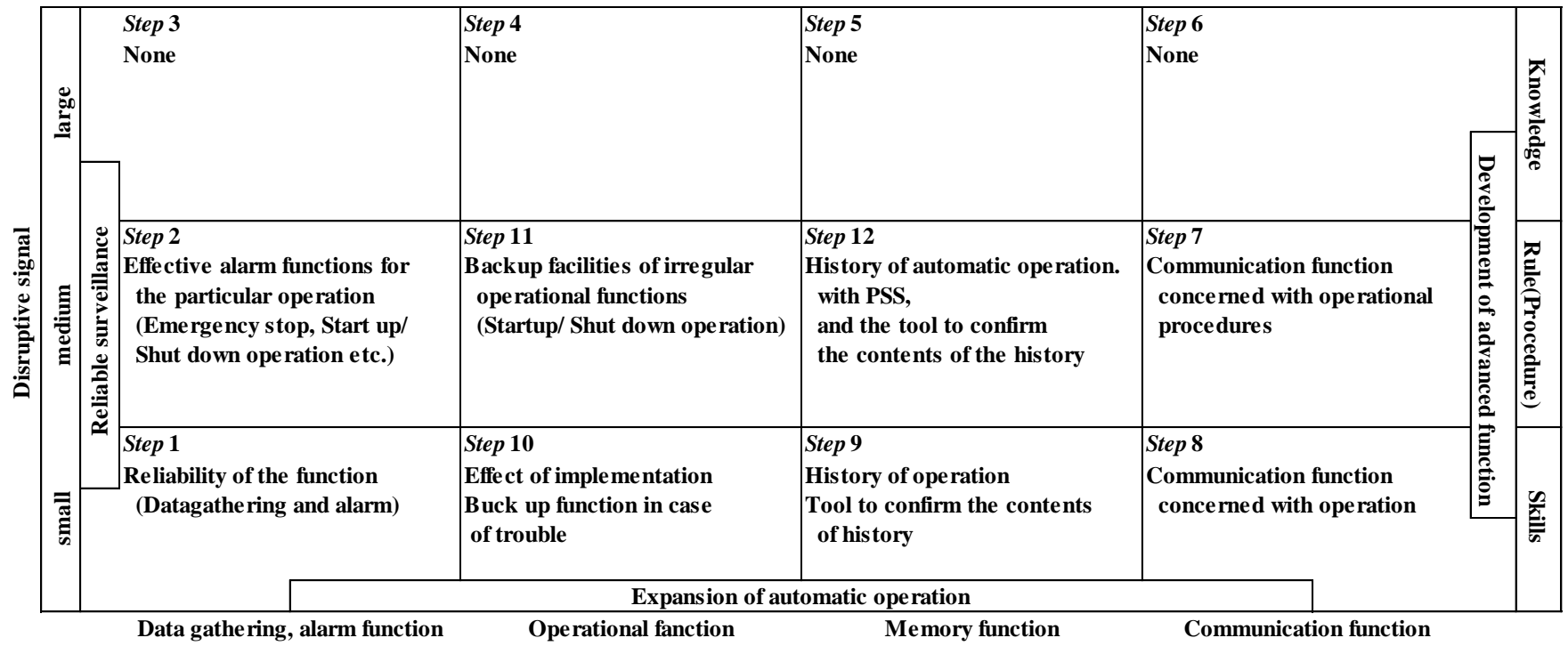

Fig. 4. Managerial components of PSS

Step 1 represents the managerial components of resilience, achieved by gathering data and by the logged alarm, used to counter a small disruptive signal. The production staff can recognize the status of a process by checking the limit of the process variables, the on/off operation of equipment and the set point change of the controller. If the alarm occurs repeatedly, the limit value of a variable is not suitable or it might be a sign of any trouble. The managerial component is the reliability of data gathering and alarm function.
Step2 represents the managerial components of resilience, fostered by data gathering and alarm function, used to counter a medium disruptive signal. At emergency stop, or start up/shut down operation, the specific alarm functions are utilized. Therefore, the managerial components are the effective alarm functions that cope with a particular operation (emergency stop or start up/shut down operation etc.).

Step3 represents the managerial components of resilience, obtained by the data gathering and alarm function used 
to counter a large disruptive signal. This function itself has no capability to restrain a large disruptive signal. Hence, there is no managerial component in this step.

Step4 represents the managerial components of resilience, provided by the operational function, used to counter a large disruptive signal. The operational function of PSS is designed to advance resource, energy and manpower savings; therefore it has no ability to restrain a large disruptive signal. Consequently, there is no managerial component in this step.

Step5 represents the managerial components of resilience, produced by the memory function, used to counter a large disruptive signal. This function is also designed to promote resource, energy and manpower savings; hence it has no ability to restrain a large disruptive signal. Therefore, there is no managerial component in this step.

Step6 represents the managerial components of resilience, achieved by the communication function, used to counter a large disruptive signal. This function is also designed to further resource, energy and manpower savings, so that it has no ability to restrain a large disruptive signal. Therefore, there is no managerial component in this step.

Step 7 represents the managerial components of resilience, produced by the communication function, used to counter a medium disruptive signal. In the case of resilience obtained by the human factors to counter a medium disruptive signal, the managerial components are the establishment of the operational procedures ${ }^{(5)}$. Therefore, the managerial components are the communication functions concerned with the operational procedures.

Step 8 represents the managerial components of resilience, provided by the communication function, used to counter a small disruptive signal. In the case of resilience produced by the human factors to counter a small disruptive signal, the managerial components are the operation of production process ${ }^{(5)}$. Hence, the managerial components are the communication functions concerned with the operation.

Step 9 represents the managerial components of resilience, yielded by the memory function, used to counter a small disruptive signal. The small disruptive signal occurs as a result of small operations. Therefore, the managerial components are the history of operation and the tools to confirm the contents of the history.

Step 10 represents the managerial components of resilience, returned by the operational function, used to counter a small disruptive signal. This function is designed to forward resource, energy and manpower savings. Hence, the managerial components are the effect of implementation and the backup function in case of any occurrences of trouble.

Step 11 represents the managerial components of resilience, produced by the operational function, used to counter a medium disruptive signal. During the automatic control of irregular operations (e.g. start up/shut down operation), the backup function is necessary to avoid the failure of operation. Therefore, the managerial components are the backup facilities for irregular operations.

Step 12 represents the managerial components of resilience, gained by the memory function, used to counter a medium disruptive signal. In the case of resilience produced by the human factors to counter a medium disruptive signal, the managerial components are the establishment of the operational procedures ${ }^{(5)}$. Therefore, the managerial components of resilience are the history of the automatic operation using a function of PSS and the tool to confirm the contents of the history.

\subsection{Specific approaches and some remarks about the managerial objectives}

The principal functions of the production support system (PSS) are the operational and memory functions. The basic functions of the PSS are implemented as formalized techniques (i.e. software algorithm) based on the skills of production staff ${ }^{(6)}$. When adding a new technique to the PSS, the most effective function is selected, taking the return on the investment into consideration ${ }^{(6)}$. It is impossible to restrain a large disruptive signal using the PSS, therefore any resilience that the PSS may produce to counter a disruptive signal is limited. But if the PSS can generate the resilience to counter small and medium scale of disruptive signals, instead of the production staff, he/she will accrue the margin of work hours to devote to the improvement of the skills and knowledge. As a result, it is possible to deal with a large disruptive signal using skills and knowledge.

\section{Conclusion}

In the chemical industry, there are two sources of resilience that restrain a disruptive signal on a production 
process.

(a) The skills and knowledge of production staff (human factors)

(b) The production units and the production support system (PSS) (artificial factors)

In this paper, the managerial components of resilience, generated by the artificial factors, were discussed using the resilience matrix that depends on the scale of a disruptive signal and on the managerial objectives. Moreover, the specific approaches and some remarks were addressed. Resilience, by restraining the influence of disruptive signals, is essential to maintain the high productivity in the production process. Human factors are the most significant components to deal with a large disruptive signal, therefore the artificial factors of the production process have to play a key role in supporting the production staff. If the PSS can reduce the workload of production staff in countering small and medium disruptive signals, the production staff can use the time gained to improve the level of skills and knowledge through training and education. As a result of this process, the resilience available to counter large-scale disruptive signals will also be improved.

\section{Acknowledgements}

This research was partially supported by the Ministry of Education, Science, Sports and Culture, Grant-in-Aid for Scientific Research (B), No.24310119 (2012) and (B), No.25282101 (2013).

\section{References}

(1) G.H.A.Shirali, M.Motamedozade, I.Mohammadfam, V.Ebrahimipour, A.Moghimbeigi, "Challenges in building resilience engineering (RE) and adaptive capacity: A field study in a chemical plant", PROCESS SAFETY AND ENVIRONMENTAL PROTECTION, Vol.99, pp. 83-90, 2012

(2) D. Furniss, J. Back, A. Blandford, M. Hilderbrandt, H. Broberg, "A resilience markers framework for small teams", Reliability Engineering and System Safety, Vol.96, pp. 2-10, 2011

(3) J. Back, D. Furniss, M. Hilderbrandt, A.Blandford, "Resilience marker for safer systems and organizations", Proceedings of Safe Computing, 2008

(4) F. Bracco, T. Piccinno, G. Dorigatti, "Turning Variability into Emergent Safety: the Resilience Matrix for Providing Strong Responses to Weak Signals", Proceedings of $5^{\mathrm{TH}}$ SYMPOSIUM ON RESILIENCE ENGINEERING MANAGING TRADE-OFF, pp.23-28, 2013

(5) H. Eguchi, T. Aoyama, K. Seki, D.O’Donovan, Ichiro Koshijima, "The impact of organizational structure on resilience based on human factors in the chemical industry", Proceedings of SOMChE 2014, PSE053, 2014

(6) H. Eguchi, "Assessment Strategy for Efficiency of Production Support System (PSS) in Chemical Factories", Kagaku Kogaku Ronbunshu, Vol. 34, pp.168-174, 2008

(7) H. Eguchi, D. O’Donovan, "Effect of a Change in Personnel on the Quantitative Evaluation of Human Skills in the Chemical Industry", J. Chem. Eng. Japan, Vol.44, pp. 32-36, 2011 